\title{
Lattice Boltzmann Simulation of Nucleate Pool Boiling in Saturated Liquid
}

\author{
Yoshito Tanaka ${ }^{1}$, Masato Yoshino ${ }^{2,3, *}$ and Tetsuo Hirata ${ }^{2}$ \\ ${ }^{1}$ Department of Mathematics and System Development Engineering, Interdisciplinary \\ Graduate School of Science and Technology, Shinshu University, 4-17-1, Wakasato, \\ Nagano-shi, Nagano 380-8553, Japan. \\ 2 Department of Mechanical Systems Engineering, Faculty of Engineering, Shinshu \\ University, Nagano-shi, Nagano 380-8553, Japan. \\ ${ }^{3}$ CREST, Japan Science and Technology Agency, 4-1-8, Honcho, Kawaguchi-shi, \\ Saitama 332-0012, Japan.
}

\begin{abstract}
A thermal lattice Boltzmann method (LBM) for two-phase fluid flows in nucleate pool boiling process is proposed. In the present method, a new function for heat transfer is introduced to the isothermal LBM for two-phase immiscible fluids with large density differences. The calculated temperature is substituted into the pressure tensor, which is used for the calculation of an order parameter representing two phases so that bubbles can be formed by nucleate boiling. By using this method, twodimensional simulations of nucleate pool boiling by a heat source on a solid wall are carried out with the boundary condition for a constant heat flux. The flow characteristics and temperature distribution in the nucleate pool boiling process are obtained. It is seen that a bubble nucleation is formed at first, and then the bubble grows and leaves the wall, finally going up with deformation by the buoyant effect. In addition, the effects of the gravity and the surface wettability on the bubble diameter at departure are numerically investigated. The calculated results are in qualitative agreement with other theoretical predictions with available experimental data.
\end{abstract}

AMS subject classifications: 76T10, 76M28, 80A22

Key words: lattice Boltzmann method (LBM), two-phase fluid flows, heat transfer, nucleate pool boiling, wettability.

\section{Introduction}

Two-phase fluid flows with phase change are of great importance in science and engineering fields. In particular, flows with nucleate boiling can be found in many industrial

*Corresponding author. Email addresses: s07t254@shinshu-u.ac.jp (Y. Tanaka), masato@shinshu-u.ac.jp (M. Yoshino), hirata@shinshu-u.ac.jp (T. Hirata) 
applications because the efficiency of heat transfer is much higher than in single phase flows. In these applications, it is necessary to make correct predictions of heat transfer characteristics of two-phase fluid flows. However, most of the past studies on such nucleate boiling were related to collection of data for boiling process obtained as a function of any of the several independent variables: heater and flow conditions, system pressure, liquid/vapor thermophysical properties, and so on (e.g., see reviews [1-3]). Thus, although the effects of these parameters on the heat transfer coefficient have been investigated and quite well established, the detailed mechanisms were not fully understood owing to its complexity. Nevertheless, with the current advance in precision measuring devices and high-performance computers, several experimental and numerical studies of these issues have recently been performed.

Main features of the nucleate boiling process that affect the rate of heat transfer during the ebullition cycle are the bubble radius at departure and the frequency at which bubbles are generated and departed. J. Kim and M. H. Kim [4] performed quantitative analyses of bubble departures during nucleate pool boiling, and obtained dimensionless scales based on experimental data that had been previously reported in many studies. With regard to numerical researches, for example, Kunugi et al. [5] carried out direct numerical simulation of pool boiling phenomena by the MARS (Multi-interface Advection and Reconstruction Solver) method [6]. Mukherjee and Kandlikar [7] used the numerical model of Mukherjee and Dhir [8] to simulate vapor bubble growth on a heated wall by means of the level-set technique.

Whereas these simulations of boiling phenomena require some models for phase change such as the enthalpy method or temperature recovery method [9], an alternative approach has been proposed to simulate nucleate boiling without using any of the models. The method is based on the free-energy approach in nonequilibrium thermodynamics. Takada and Tomiyama [10] conducted numerical simulation of two-phase flows with phase change using a phase-field method. Seta and Okui [11] proposed a lattice Boltzmann method (LBM) $[12,13]$ with heat transfer to simulate pool boiling in two-dimensional flows. More recently, Hazi and Markus [14] also carried out lattice Boltzmann simulations of pool boiling phenomena, and investigated bubble diameter at departure and release frequency under different gravity and various wetting conditions on a heated surface.

In the LBM for two-phase fluid flows, several models [15-18] have been developed and successfully applied to many kinds of issues such as phase separations, instability problems, droplet dynamics, bubble flows, and so on. In particular, Inamuro et al. [19] proposed two-phase LBM, which enables the stable calculation of two-phase fluid flows with large density ratios of up to 1000 . In the present paper, therefore, we incorporate the effects of heat transfer with nucleate boiling into the two-phase LBM proposed by Inamuro et al. to simulate the nucleate pool boiling on a heated surface. By using this method, the effects of the gravity and the surface wettability on the bubble diameter at departure are numerically investigated. 


\section{Numerical method}

\subsection{Two-phase lattice Boltzmann method}

Non-dimensional variables, which are defined by using a characteristic length $L_{0}$, a characteristic particle speed $c$, a characteristic time scale $t_{0}=\sqrt{L_{0} / g_{0}}$, where $g_{0}$ is the gravitational acceleration, a reference order parameter $\phi_{0}$, and a reference density $\rho_{0}$ are used as in [19]. In the LBM, a modeled fluid, composed of identical particles whose velocities are restricted to a finite set of $N$ vectors $c_{i}(i=1,2,3, \ldots, N)$, is considered. The nine-velocity model $(N=9)$ given by the following equation is used in the computations:

$$
\left[c_{1}, c_{2}, c_{3}, c_{4}, c_{5}, c_{6}, c_{7}, c_{8}, c_{9}\right]=\left[\begin{array}{rrrrrrrrr}
0 & 1 & 0 & -1 & 0 & 1 & -1 & -1 & 1 \\
0 & 0 & 1 & 0 & -1 & 1 & 1 & -1 & -1
\end{array}\right]
$$

The physical space is divided into a square lattice, and the evolution of particle population at each lattice site is computed. Two particle velocity distribution functions, $f_{i}$ and $g_{i}$, are used. The function $f_{i}$ is used for the calculation of an order parameter which represents two phases, and the function $g_{i}$ is used for the calculation of a predicted velocity of the two-phase fluid without a pressure gradient. The evolution of the particle distribution functions $f_{i}(\boldsymbol{x}, t)$ and $g_{i}(\boldsymbol{x}, t)$ with velocity $\boldsymbol{c}_{i}$ at the point $\boldsymbol{x}$ and at time $t$ is computed by the following equations:

$$
\begin{aligned}
& f_{i}\left(x+c_{i} \Delta x, t+\Delta t\right)=f_{i}^{c}(x, t), \\
& g_{i}\left(x+c_{i} \Delta x, t+\Delta t\right)=g_{i}^{\mathrm{c}}(x, t),
\end{aligned}
$$

where $f_{i}^{\mathrm{c}}$ and $g_{i}^{\mathrm{c}}$, given below, are functions in which variables $x$ and $t$ enter only through macroscopic variables and/or their derivatives, $\Delta x$ is a spacing of the square lattice, and $\Delta t$ is a time step during which the particles travel the lattice spacing.

The order parameter $\phi$ distinguishing two phases and the predicted velocity $\boldsymbol{u}^{*}$ of the two-phase fluids are defined as follows:

$$
\begin{aligned}
\phi & =\sum_{i=1}^{9} f_{i} \\
\boldsymbol{u}^{*} & =\sum_{i=1}^{9} \boldsymbol{c}_{i} g_{i} .
\end{aligned}
$$


The functions $f_{i}^{c}$ and $g_{i}^{c}$ in the Eqs. (2.2) and (2.3) are given as follows:

$$
\begin{aligned}
f_{i}^{c}= & H_{i} \phi+F_{i}\left[p_{0}-\kappa_{f} \phi \frac{\partial^{2} \phi}{\partial x_{\alpha}^{2}}\right]+3 E_{i} \phi c_{i \alpha} u_{\alpha}+E_{i} \kappa_{f} G_{\alpha \beta}(\phi) c_{i \alpha} c_{i \beta}+E_{i} C \frac{\partial P_{\alpha \beta}}{\partial x_{\beta}} c_{i \alpha}, \\
g_{i}^{c}= & E_{i}\left[1+3 c_{i \alpha} u_{\alpha}-\frac{3}{2} u_{\alpha} u_{\alpha}+\frac{9}{2} c_{i \alpha} c_{i \beta} u_{\alpha} u_{\beta}+\frac{3}{4} \Delta x\left(\frac{\partial u_{\beta}}{\partial x_{\alpha}}+\frac{\partial u_{\alpha}}{\partial x_{\beta}}\right) c_{i \alpha} c_{i \beta}\right. \\
& \left.+3 c_{i \alpha} \frac{\Delta x}{\rho} \frac{\partial}{\partial x_{\beta}}\left\{\mu\left(\frac{\partial u_{\beta}}{\partial x_{\alpha}}+\frac{\partial u_{\alpha}}{\partial x_{\beta}}\right)\right\}\right]+E_{i} \frac{\kappa_{g}}{\rho} G_{\alpha \beta}(\rho) c_{i \alpha} c_{i \beta} \\
& -\frac{1}{2} F_{i} \frac{\kappa_{g}}{\rho}\left(\frac{\partial \rho}{\partial x_{\alpha}}\right)^{2}-3 E_{i} c_{i y}\left(1-\frac{\rho_{\mathrm{L}}}{\rho}\right) g \Delta x+3 E_{i} c_{i y} \frac{g \beta_{\theta}}{\rho}\left(\theta-\theta^{*}\right) \Delta x
\end{aligned}
$$

where

$$
\begin{aligned}
p_{0} & =\frac{\phi T}{1-b \phi}-a \phi^{2} \\
G_{\alpha \beta}(\phi) & =\frac{9}{2} \frac{\partial \phi}{\partial x_{\alpha}} \frac{\partial \phi}{\partial x_{\beta}}-\frac{9}{4} \frac{\partial \phi}{\partial x_{\gamma}} \frac{\partial \phi}{\partial x_{\gamma}} \delta_{\alpha \beta} \\
P_{\alpha \beta} & =\left[p_{0}-\kappa_{f} \phi \frac{\partial^{2} \phi}{\partial x_{\gamma}^{2}}-\frac{\kappa_{f}}{2}\left(\frac{\partial \phi}{\partial x_{\gamma}}\right)^{2}\right] \delta_{\alpha \beta}+\kappa_{f} \frac{\partial \phi}{\partial x_{\alpha}} \frac{\partial \phi}{\partial x_{\beta}}
\end{aligned}
$$

and

$$
\left.\begin{array}{l}
H_{1}=1, \quad H_{2}=H_{3}=H_{4}=\cdots=H_{9}=0, \\
F_{1}=-5 / 3, \quad F_{i}=3 E_{i}(i=2,3,4, \ldots, 9), \\
E_{1}=4 / 9, \quad E_{2}=E_{3}=E_{4}=E_{5}=1 / 9, \\
E_{6}=E_{7}=E_{8}=E_{9}=1 / 36 .
\end{array}\right\}
$$

with $\alpha, \beta, \gamma=x, y$ (subscripts $\alpha, \beta$, and $\gamma$ represent Cartesian coordinates and the summation convention is used). Note that the subscripts $\mathrm{L}$ and $\mathrm{G}$ indicate liquid and gas phases, respectively. In the above equations, $u_{\alpha}, \rho$, and $\mu$ are the velocity, density, and viscosity of two-phase fluids, respectively; $g$ is the gravitational acceleration acting in the $-y$ direction; $\delta_{\alpha \beta}$ is the Kronecker delta; $\kappa_{f}$ and $\kappa_{g}$ are constant parameters determining the width of the interface and the strength of the interfacial tension $\sigma$, respectively; $\beta_{\theta}$ is the volumetric expansion coefficient; $\theta$ and $\theta^{*}$ are the temperature and the reference temperature, respectively; $a, b$, and $T$ are free parameters determining the maximum and minimum values of $\phi$; and $C$, which is of $O(1)$, is a constant parameter related to the mobility $\theta_{\mathrm{M}}$ as follows:

$$
\theta_{\mathrm{M}}=\left(\frac{1}{2}-\frac{1}{3} C\right) \Delta x
$$


The first and second derivatives (divergence) in Eqs. (2.6), (2.7), (2.9), and (2.10) are calculated by using the following finite difference approximations:

$$
\begin{aligned}
\frac{\partial \psi}{\partial x_{\alpha}} & =\frac{1}{6 \Delta x} \sum_{i=2}^{9} c_{i \alpha} \psi\left(x+c_{i} \Delta x\right) \\
\frac{\partial^{2} \psi}{\partial x_{\alpha}^{2}} & =\frac{1}{3(\Delta x)^{2}}\left[\sum_{i=2}^{9} \psi\left(x+c_{i} \Delta x\right)-8 \psi(x)\right] .
\end{aligned}
$$

The density in the interface is obtained by using the cut-off values of the order parameter, $\phi_{\mathrm{L}}^{*}$ and $\phi_{\mathrm{G}}^{*}$, for the liquid and gas phases with the following relation:

$$
\rho= \begin{cases}\rho_{\mathrm{G}} \\ \frac{\rho_{\mathrm{L}}-\rho_{\mathrm{G}}}{2}\left\{\sin \left[\frac{\phi-\left(\phi_{\mathrm{L}}^{*}+\phi_{\mathrm{G}}^{*}\right) / 2}{\phi_{\mathrm{L}}^{*}-\phi_{\mathrm{G}}^{*}}\right]+1\right\}+\rho_{\mathrm{G}}, & \phi<\phi_{\mathrm{G}}^{*} \leq \phi \leq \phi_{\mathrm{L}}^{*} . \\ \rho_{\mathrm{L}}, & \phi_{\mathrm{L}}^{*}<\phi\end{cases}
$$

The viscosity $\mu$ in the interface is obtained by the following equation:

$$
\mu=\frac{\rho-\rho_{\mathrm{G}}}{\rho_{\mathrm{L}}-\rho_{\mathrm{G}}}\left(\mu_{\mathrm{L}}-\mu_{\mathrm{G}}\right)+\mu_{\mathrm{G}} .
$$

The interfacial tension $\sigma$ is given by

$$
\sigma=\kappa_{g} \int_{-\infty}^{\infty}\left(\frac{\partial \rho}{\partial \xi}\right)^{2} d \xi
$$

where $\xi$ indicates the coordinate normal to the interface [20].

Since $\boldsymbol{u}^{*}$ is not divergence free $\left(\nabla \cdot \boldsymbol{u}^{*} \neq 0\right)$ in general, it is required for $\boldsymbol{u}^{*}$ to be corrected by using the Poisson equation for the pressure $p$ of the two-phase fluids. By using the explicit Euler method for the temporal integration, the Navier-Stokes equation is expressed as

$$
S h \frac{\boldsymbol{u}^{n+1}-\boldsymbol{u}^{n}}{\Delta t}+\left(\boldsymbol{u}^{n} \cdot \nabla\right) \boldsymbol{u}^{n}=-\frac{\nabla p}{\rho}+\frac{1}{\operatorname{Re}} \nabla^{2} \boldsymbol{u}^{n}+\boldsymbol{F},
$$

where $S h=U / c$ is the Strouhal number, $\boldsymbol{F}$ is the external force term (the surface tension and buoyancy terms), and the superscript $n$ indicates the value at $t=n \Delta t$. Eq. (2.18) is divided into the following two equations:

$$
\begin{aligned}
S h \boldsymbol{u}^{*} & =S h \boldsymbol{u}^{n}+\left[\frac{1}{R e} \nabla^{2} \boldsymbol{u}^{n}-\left(\boldsymbol{u}^{n} \cdot \nabla\right) \boldsymbol{u}^{n}+\boldsymbol{F}\right] \Delta t, \\
\operatorname{Sh} \frac{\boldsymbol{u}^{n+1}-\boldsymbol{u}^{*}}{\Delta t} & =-\frac{\nabla p}{\rho} .
\end{aligned}
$$


Taking the divergence of Eq. (2.20) and considering the continuity equation $\left(\nabla \cdot \boldsymbol{u}^{n+1}=0\right)$, we get the following Poisson equation for the pressure $p$ of the two-phase fluids:

$$
\nabla \cdot\left(\frac{\nabla p}{\rho}\right)=\operatorname{Sh} \frac{\nabla \cdot \boldsymbol{u}^{*}}{\Delta t}
$$

In order to solve Eq. (2.21), a new velocity distribution function $h_{i}$ is introduced [19], and the following evolution equation of $h_{i}$ is used for the calculation of $p$ :

$$
h_{i}^{m+1}\left(x+c_{i} \Delta x\right)=h_{i}^{m}(x)-\frac{1}{\tau_{h}}\left[h_{i}^{m}(x)-E_{i} p^{m}(x)\right]-\frac{1}{3} E_{i} \frac{\partial u_{\alpha}^{*}}{\partial x_{\alpha}} \Delta x,
$$

where $m$ is the number of iterations and the relaxation time $\tau_{h}$ is give by

$$
\tau_{h}=\frac{1}{\rho}+\frac{1}{2}
$$

The pressure $p$ is obtained by

$$
p=\sum_{i=1}^{9} h_{i}
$$

The calculation of Eq. (2.22) is iterated until $\left|p^{m+1}-p^{m}\right|<\varepsilon$ is satisfied in the whole domain. The tolerance $\varepsilon$ is set to $1.0 \times 10^{-6}$ in the following calculations.

The temperature $\theta$ of the fluids is calculated by the following equation:

$$
\theta(\boldsymbol{x}, t+\Delta t)=\sum_{i=1}^{9} T_{i}^{\mathcal{C}}\left(\boldsymbol{x}-\boldsymbol{c}_{i} \Delta x, t\right),
$$

where the function $T_{i}^{\mathrm{c}}$ is expressed as follows:

$$
T_{i}^{\mathrm{c}}=E_{i} \theta\left(1+3 c_{i \alpha} u_{\alpha}\right)+E_{i} B \Delta x c_{i \alpha} \frac{\partial \theta}{\partial x_{\alpha}},
$$

where $B$ is a parameter determining the thermal diffusivity $\alpha_{\theta}$ and the thermal conductivity $\lambda$. The relations between these parameters are as follows:

$$
\begin{aligned}
\alpha_{\theta} & =\left(\frac{1}{6}-\frac{1}{3} B\right) \Delta x \\
\lambda & =\left(\frac{1}{3}-\frac{1}{3} B\right) \Delta x .
\end{aligned}
$$

Furthermore, the parameter $B$ in the interface is given by

$$
B=\frac{\rho-\rho_{\mathrm{G}}}{\rho_{\mathrm{L}}-\rho_{\mathrm{G}}}\left(B_{\mathrm{L}}-B_{\mathrm{G}}\right)+B_{\mathrm{G}} .
$$




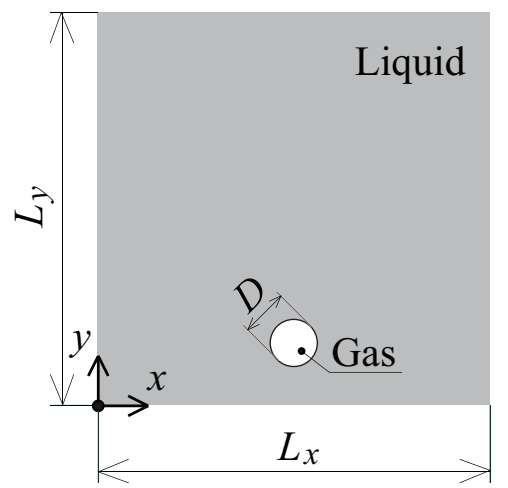

Figure 1: Computational domain of a single bubble rising in saturated liquid.

The calculated temperature $\theta$ is substituted for $T$ in the pressure tensor (2.8). Consequently, the value of $f_{i}^{c}$ is changed by the temperature, and bubbles can be formed by the boiling.

Applying the asymptotic theory [21] to Eqs. (2.2), (2.3), (2.22), and (2.25), we find that the asymptotic expansions of macroscopic variables, $\phi, \rho, u, p$, and $\theta$, satisfy the phasefield advection-diffusion equation (the Cahn-Hilliard equation with advection) for $\phi$, the continuity equation, the Navier-Stokes equations for incompressible two-phase fluid including the interfacial tension and the buoyancy, and the convection-diffusion equations for the temperature with relative errors of $O\left[(\Delta x)^{2}\right]$ [22].

\subsection{Wetting boundary condition}

Recently, Briant et al. $[23,24]$ have proposed a wetting boundary condition which enables the contact angle of the interface to be controlled in a way consistent with Cahn theory [25]. In their method, the derivative of the density normal to the wall, $\partial \rho / \partial n$, is specified by using the wetting potential that is calculated according to a prescribed static contact angle $\theta_{\mathrm{S}}$. In the following computations, the specified value of $\partial \rho / \partial n$ at wall sites is substituted for the first and second derivatives in Eq. (2.6).

\section{Results and discussion}

\subsection{A single bubble rising in saturated liquid}

In order to confirm the mass conservation of the present method, the simulation of a single bubble rising in a saturated liquid is carried out. The computational domain with $L_{x}=L_{y}=200 \Delta x$ is considered, as shown in Fig. 1. A single bubble with diameter $D=16 \Delta x$ is placed in a saturated liquid at the initial state. The periodic boundary condition is used in the $x$ - and $y$-directions. The parameters determining the maximum and minimum values of the order parameter are $a=1, b=1, T=2.93 \times 10^{-1}$; it follows that these values 


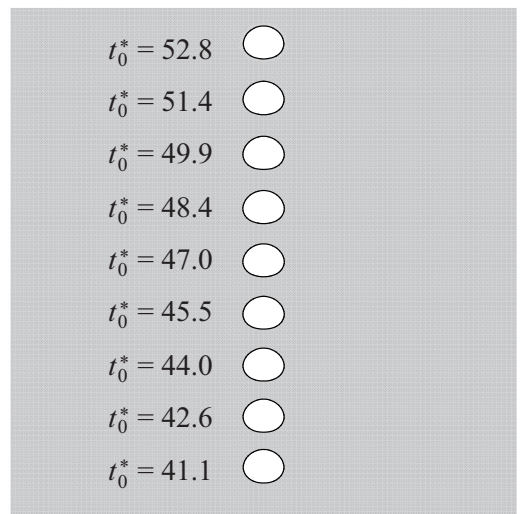

Figure 2: Time evolution of the bubble shape for $E=6.66$ and $M=18.4$. The dimensionless time is $t_{0}^{*}=$ $t v_{\mathrm{t}} / D_{\mathrm{e}}$, where $v_{\mathrm{t}}$ and $D_{\mathrm{e}}$ are the terminal velocity and the equivalent diameter of bubble, respectively.

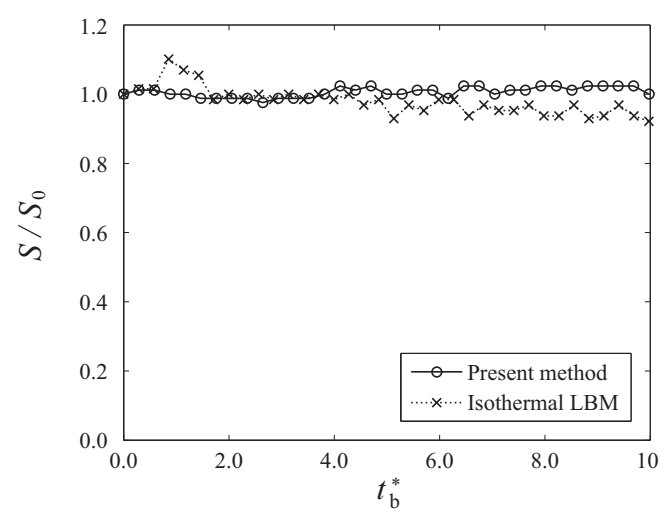

Figure 3: Time variation of mass of bubble. The area of mass, $S$, is normalized by the value when calculation starts. The dimensionless time is $t_{\mathrm{b}}^{*}=$ $\left(t-t_{\mathrm{b}}\right) v_{\mathrm{t}} / D_{\mathrm{e}}$, where $v_{\mathrm{t}}, D_{\mathrm{e}}$, and $t_{\mathrm{b}}$ are the terminal velocity, the equivalent diameter of bubble, and the beginning time of calculation, respectively.

are $\phi_{\max }=4.031 \times 10^{-1}$ and $\phi_{\min }=2.638 \times 10^{-1}$, respectively. The cut-off values of the order parameter for obtaining the density in the interface are $\phi_{\mathrm{L}}^{*}=3.80 \times 10^{-1}$ and $\phi_{\mathrm{G}}^{*}=$ $2.75 \times 10^{-1}$. The other computational parameters are as follows: $\rho_{\mathrm{L}}=50, \rho_{\mathrm{G}}=1, \mu_{\mathrm{L}}=$ $1.0 \times 10^{-1} \Delta x, \mu_{\mathrm{G}}=1.0 \times 10^{-2} \Delta x, \theta_{\mathrm{L}}=\theta_{\mathrm{G}}=2.93 \times 10^{-1}, \kappa_{f}=0.05(\Delta x)^{2}, \kappa_{g}=1.0 \times 10^{-8}(\Delta x)^{2}$, $g \Delta x=4.0 \times 10^{-9}, \theta_{\mathrm{M}}=1.0 \times 10^{-2} \Delta x, \lambda_{\mathrm{L}}=\lambda_{\mathrm{G}}=1.7 \times 10^{-1} \Delta x, \alpha_{\theta, \mathrm{L}}=\alpha_{\theta, \mathrm{G}}=1.0 \times 10^{-6} \Delta x$, and $\beta_{\theta, \mathrm{L}}=\beta_{\theta, \mathrm{G}}=1.0 \Delta x$. The dimensionless numbers for this problem are Eötvös number $E=$ $g D^{2}\left(\rho_{\mathrm{L}}-\rho_{\mathrm{G}}\right) / \sigma$ and Morton number $M=g \mu_{\mathrm{L}}^{4}\left(\rho_{\mathrm{L}}-\rho_{\mathrm{G}}\right) /\left(\rho_{\mathrm{L}}^{2} \sigma^{3}\right)$; their values are $E=6.66$ and $M=18.4$.

Fig. 2 shows the time evolution of the bubble shape for $E=6.66$ and $M=18.4$. From this figure, it is seen that the bubble rises in a saturated liquid with its oblate ellipsoidal shape maintained. The simulated bubble shape agrees with the shape in the experimental regime map [26]. Fig. 3 shows the time variation of the mass of the bubble. After the simulation is executed and the flow field is fully developed, calculation of the mass of the bubble is started. The mass of the bubble, $S$, is calculated by counting the number of nodes inside the interface, $\left(\rho_{\mathrm{L}}+\rho_{\mathrm{G}}\right) / 2$. In addition, $S$ is normalized by the value at the beginning time of the calculation. It is noted that the dimensionless time is $t_{\mathrm{b}}^{*}=$ $\left(t-t_{\mathrm{b}}\right) v_{\mathrm{t}} / D_{\mathrm{e}}$, where $t_{\mathrm{b}}$ is the beginning time of the calculation. From this figure, it is seen that the mass of the bubble is conserved during the calculation in spite of the small diameter $(D=16 \Delta x)$. Hence, the mass conservation of the bubble by the present method verified and comparable to the result by the conventional isothermal LBM.

\subsection{Estimation of domain size}

Next, preliminary simulations of a nucleate pool boiling are carried out in order to estimate the size of the domain. We consider a rectangular domain with fixed $L_{y}=200 \Delta x$ and 


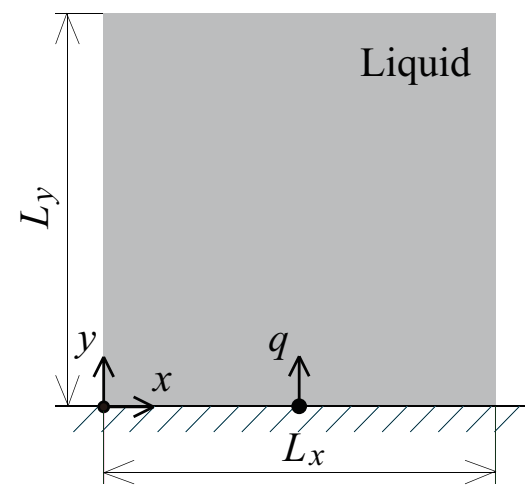

Figure 4: Computational domain of nucleate pool boiling.

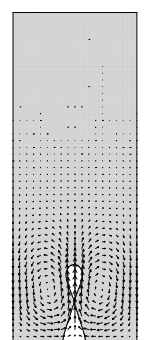

(a)

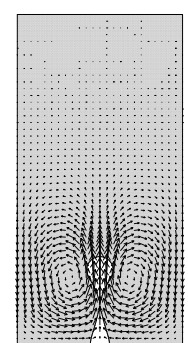

(b)

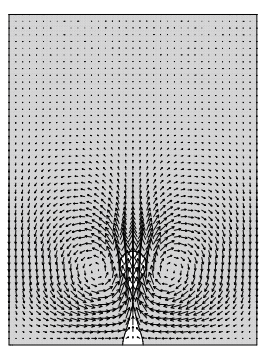

(c)

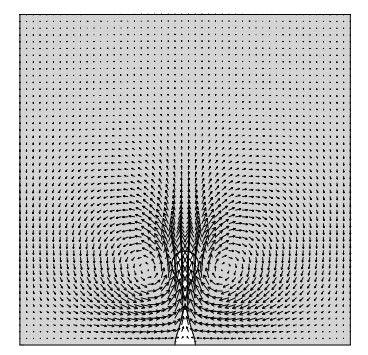

(d)

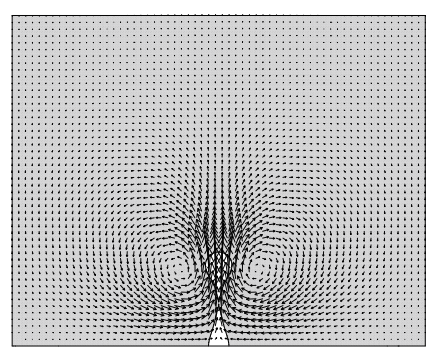

(e)

Figure 5: Bubble shape and velocity vectors for various distances among the heat sources just after detachment of the bubble: (a) $L_{x}=75 \Delta x$; (b) $L_{x}=100 \Delta x$; (c) $L_{x}=150 \Delta x$; (d) $L_{x}=200 \Delta x$; (e) $L_{x}=250 \Delta x$.

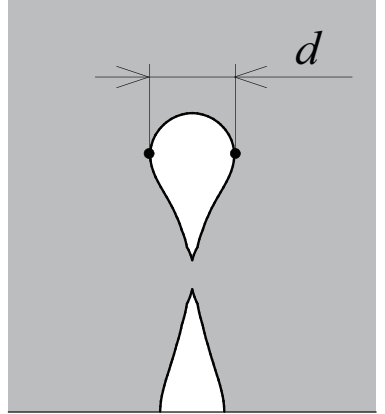

Figure 6: Definition of the bubble diameter at departure $d$. The solid line indicates the contour line of the density with $\rho=\left(\rho_{\mathrm{L}}+\rho_{\mathrm{G}}\right) / 2$.

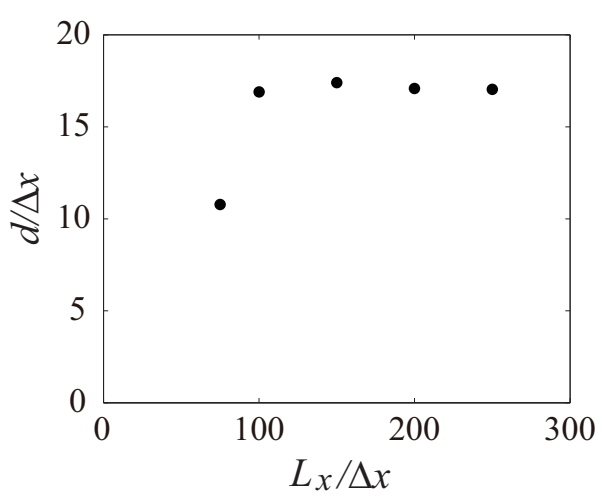

Figure 7: Bubble diameter at departure for various distances among heat sources.

various values of $L_{x}\left(L_{x}=75 \Delta x, 100 \Delta x, 150 \Delta x, 200 \Delta x\right.$, and $\left.250 \Delta x\right)$, as shown in Fig. 4 . The no-slip boundary condition and the wetting boundary condition, in which the static contact angle is set to $\theta_{S}=90^{\circ}$, are applied to the bottom wall $(y=0)$. In addition, a heat source with a constant heat flux $q=5.0 \times 10^{-6}$ is placed at the central point on the bottom boundary [27] in order to compare with the theoretical predictions [28] described later. At the 


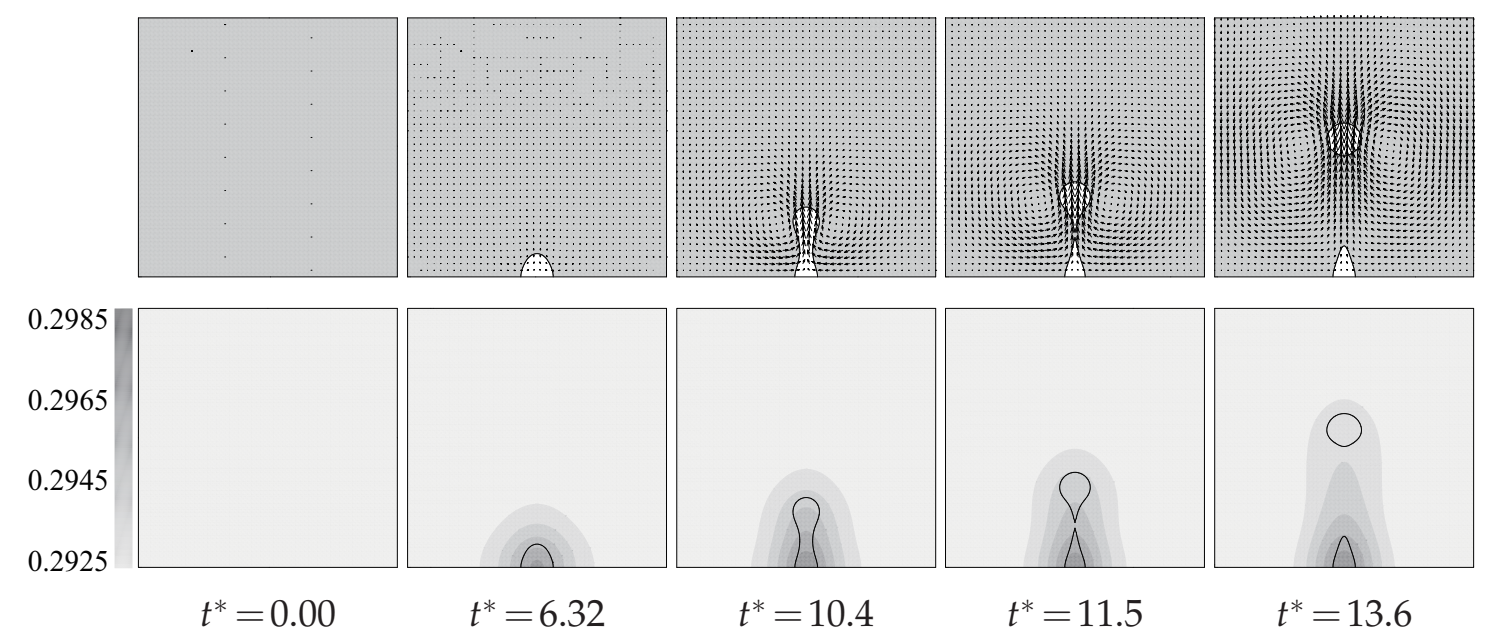

Figure 8: Time evolution of bubble shape with velocity vectors (upper) and temperature distribution (lower) for $g \Delta x=2.0 \times 10^{-9}$. The solid line indicates the contour line of the density with $\rho=\left(\rho_{\mathrm{L}}+\rho_{\mathrm{G}}\right) / 2$. The dimensionless time is $t^{*}=t / \sqrt{L_{y} / g}$.

top boundary $\left(y=L_{y}\right)$, the normal derivatives of the order parameter, the density, and the velocity are set to zero. The periodic boundary condition is used in the $x$-direction, so that the distance among the heat sources is different in each case. At the initial state, the computational domain is filled with the liquid whose temperature is $\theta_{\mathrm{L}}=2.93 \times 10^{-1}$. The other computational parameters are the same as those in the previous problem.

Fig. 5 shows the computational results of the bubble shape and velocity vectors for various distances among the heat sources just after detachment of the bubble. In all cases, circular flows can be observed on both sides of the bubble. It is seen that when the distance is over $200 \Delta x$, there is little influence on the neighboring heat sources. Furthermore, the bubble diameter at departure as defined in Fig. 6 is calculated and shown in Fig. 7. From this figure, we can see that the bubble diameter decreases when the distance is less than $100 \Delta x$. For these reasons, $L_{x}$ is set to $200 \Delta x$ in the following simulations.

\subsection{Nucleate pool boiling for various gravitational accelerations}

In order to investigate the effect of the gravity, the simulations of nucleate pool boiling for various gravitational accelerations are performed. The square domain with $L_{x}=L_{y}=$ $200 \Delta x$ is considered. In these simulations, the gravitational acceleration is changed in the range of $10^{-9}<g \Delta x<10^{-8}$. The boundary conditions and other computational parameters are the same as those in the previous problem. Fig. 8 shows the time evolution of the bubble shape with velocity vectors and temperature distributions for $g \Delta x=2.0 \times 10^{-9}$. It is seen that a bubble nucleation is formed at first, and then the bubble grows and leaves the wall, finally going up with deformation by the buoyancy. From the time evolution of velocity vectors, it is also found that circular flows appear on both sides of the bubble, 


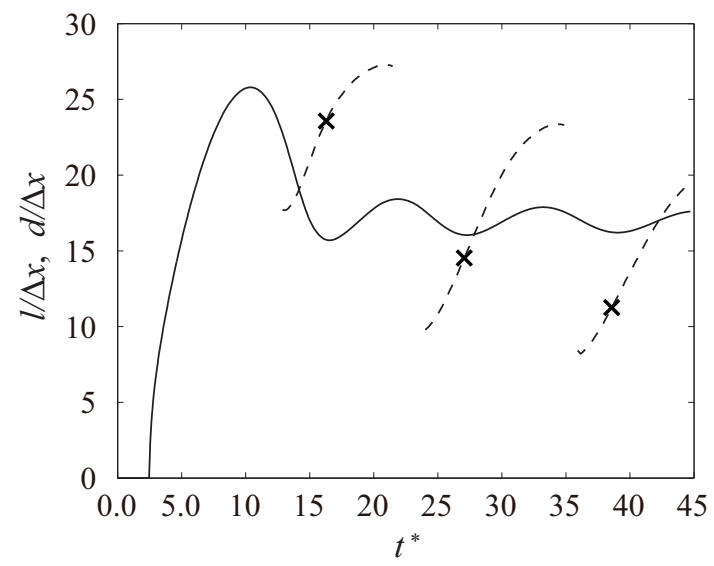

Figure 9: Time variation of characteristic lengths of bubble for $g \Delta x=2.0 \times 10^{-9}$ : - , contact length of bubble on the wall, $l ;---$, largest diameter of bubble cap, $d ; \times$, bubble diameter at departure. The dimensionless time is $t^{*}=t / \sqrt{L_{y} / g}$.

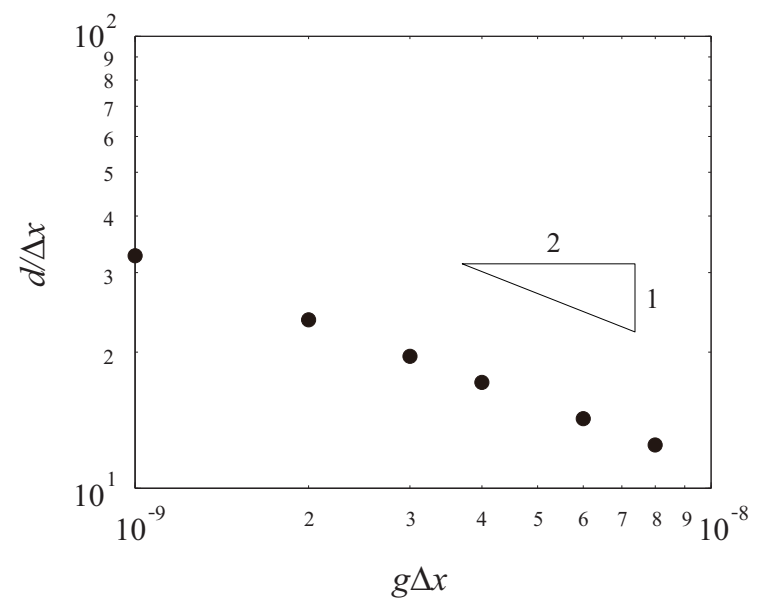

Figure 10: Bubble diameter at first departure for various gravitational accelerations.

and grow with time. After the bubble is detached, these circular flows go up with the bubble. Also, from the time evolution of temperature distribution, it is seen that heat is transferred upward by the rising bubble. Fig. 9 shows the time variations of the contact length $l$ of the bubble on the wall and the largest diameter $d$ of the bubble cap for $g \Delta x=2.0 \times 10^{-9}$. In this figure, the cross indicates the detachment of the bubble, and each dashed line is plotted during the time when the bubble stays in the computational domain after the deformation. It is seen that the value of the contact length oscillates around $17 \Delta x$ after the first detachment of the bubble. In addition, the diameter of the bubble cap increases as the contact length locally decreases. After that, the bubble cap is detached when the contact length on the wall reaches the local minimal value.

Fig. 10 shows the bubble diameter at first departure plotted against gravitational ac- 


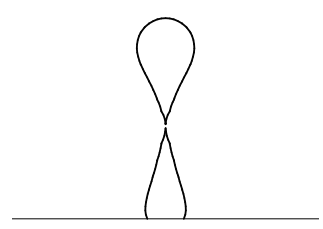

(a)

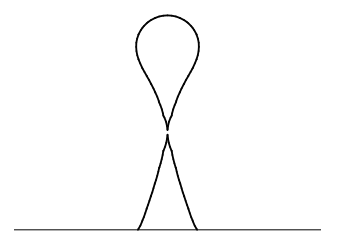

(b)

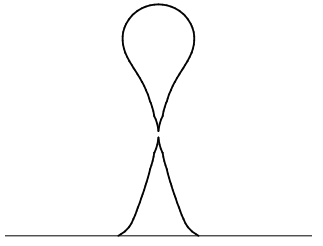

(c)

Figure 11: Bubble shape for various static contact angles just after detachment of the bubble: (a) $\theta_{\mathrm{S}}=79.5^{\circ}$; (b) $\theta_{\mathrm{S}}=107^{\circ}$; (c) $\theta_{\mathrm{S}}=140^{\circ}$.

celerations. It is seen that the bubble diameter at departure decreases with increasing gravitational accelerations. Fritz [28] investigated the bubble diameter at departure theoretically and experimentally. From his results of the experiments performed by using $\mathrm{H}_{2} \mathrm{O}, \mathrm{Hg}$, and $\mathrm{CCl}_{4}$, he concluded that the bubble diameter at departure $d$ satisfies the following relation:

$$
d \sim \theta_{\mathrm{S}} \sqrt{\frac{\sigma}{g\left(\rho_{\mathrm{L}}-\rho_{\mathrm{G}}\right)}} .
$$

It is noted that the applicable range of the above relationship is from low to middle heat fluxes. According to the Eq. (3.1), the bubble diameter at departure is proportional to $g^{-0.5}$. It is obtained from the present results that the slope of the bubble diameter at departure is nearly -0.5 over the range of the calculated gravitational acceleration. Hence, the relation between the bubble diameter at departure and gravitational acceleration is found to be reasonable.

\subsection{Nucleate pool boiling for various static contact angles}

In order to investigate the effect of wettability on the wall, the simulations of nucleate pool boiling for various static contact angles are carried out. The static contact angle is changed in the range of $79.5^{\circ} \leq \theta_{S} \leq 140^{\circ}$. The gravitational acceleration is fixed $g \Delta x=$ $6.0 \times 10^{-9}$. The boundary conditions and other computational parameters are the same as those in the previous problem. Fig. 11 shows the bubble shape for various static contact angles just after detachment of the bubble. It is seen that the contact length of the bubble on the wall spreads with increasing the static contact angle. It is also found that the bubble diameter at departure increases as the static contact angle increases.

Finally, the computational results of the bubble diameter at departure are plotted against static contact angles in Fig. 12. It is seen that the bubble diameter at departure increases almost linearly in the range of the present static contact angle. In Eq. (3.1), the bubble diameter at departure is proportional to the static contact angle. Therefore, the relation between the bubble diameter at departure and the static contact angle is in good agreement with other theoretical predictions with available experimental data. In con- 


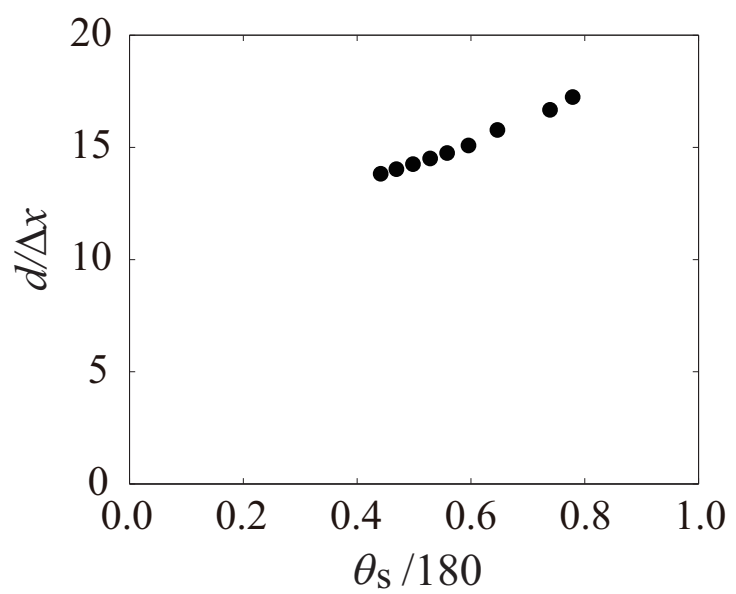

Figure 12: Bubble diameter at departure for various static contact angles.

trast, the computational results of the bubble diameter at departure deviates from this linearity in the range of $\theta_{S}<79.5^{\circ}$, because the size of the heat source is too small in these simulations. Thus, simulations with higher resolution are required for the nucleate pool boiling on the hydrophilic wall.

\section{Concluding remarks}

A numerical method for nucleate pool boiling based on the lattice Boltzmann method for two-phase fluids with large density differences has been proposed. The method was applied to two-dimensional simulations of nucleate pool boiling by a heat source on a solid wall. By comparing the present results to theoretical predictions with available experimental data regarding the bubble diameter at departure, it is found that the present method yields qualitatively reasonable results.

It is found that in preliminary calculations by the present method, we can simulate the nucleate pool boiling for water-vapor systems at 373K (the density ratio is about 1600 ) by a heat source with a constant temperature, though the cost of these simulations is more expensive. In general, spurious velocities for high density ratio become larger than those for low density ratio. However, the spurious velocities don't affect the behavior of the nucleate pool boiling, since they are smaller than the fluid velocities due to the gravity and the temperature difference. In this simulation, the ratio of the maximum spurious velocity to the maximum fluid velocity when the bubble is detached is $6.6 \%$, where the spurious velocity is estimated by calculating a stationary spherical droplet whose diameter is the same as the equivalent diameter converted from the area of the detached bubble. The spurious velocities can be reduced with decreasing the interfacial tension and with increasing the lattice points in the interface, as reported by Inamuro et al. [19]. In addition, three-dimensional simulations are needed for quantitative investiga- 
tions, and in particular, the LBM has a great advantage of extension to three-dimension in a straightforward way. Thus, three-dimensional simulations for high density ratio with high resolution are required in future work.

\section{Acknowledgments}

This work was partly supported by the Grant-in-Aid for Young Scientists (B) [No.18760121] of the Ministry of Education, Culture, Sports, Science and Technology (MEXT) in Japan.

\section{References}

[1] R. Mei, W. Chen, and J. F. Klausner, Vapor bubble growth in heterogeneous boiling-I. Formulation, Int. J. Heat Mass Transfer, 38 (1995), 909-919.

[2] V. H. Dhir, Numerical simulations of pool-boiling heat transfer, AIChE J., 47 (2001), 813-834.

[3] I. L. Pioro, W. Rohsenow, and S. S. Doerffer, Nucleate pool-boiling heat transfer. I: review of parametric effects of boiling surface, Int. J. Heat Mass Transfer, 47 (2004), 5033-5044.

[4] J. Kim and M. H. Kim, On the departure behaviors of bubble at nucleate pool boiling, Int. J. Multiphase Flow, 32 (2006), 1269-1286.

[5] T. Kunugi, N. Saito, T. Fujita, and A. Serizawa, Direct numerical simulation of pool and forced convective flow boiling phenomena, Proc. The 12th Int. Heat Transfer Conf., (2002), 497-502.

[6] T. Kunugi, MARS for multiphase calculation, Comput. Fluid Dyn. J., 9 (2001), 563-571.

[7] A. Mukherjee and S. G. Kandlikar, Numerical study of single bubbles with dynamic contact angle during nucleate pool boiling, Int. J. Heat Mass Transfer, 50 (2007), 127-138.

[8] A. Mukherjee and V. K. Diher, Study of lateral merger of vapor bubbles during nucleate pool boiling, J. Heat Transfer, 126 (2004), 1023-1039.

[9] Ohnaka, I., Introduction to Computational Analysis of Heat Transfer and Solidification Application to the Casting Processes-, Maruzen, (1985) 202 [in Japanese].

[10] N. Takada and A. Tomiyama, Interface-tracking simulation of two-phase flows by phasefield method, ASME Joint U.S.-European Fluids Eng. Summer Meeting, (2006), Paper No. FEDSM2006-98536.

[11] T. Seta and K. Okui, The single component thermal lattice Boltzmann simulation of pool boiling in two dimensions, J. Therm. Sci. Technol., 1 (2006), 125-137.

[12] S. Chen and G. D. Doolen, Lattice Boltzmann method for fluid flows, Annu. Rev. Fluid Mech., 30 (1998), 329-364.

[13] S. Succi, The Lattice Boltzmann Equation for Fluid Dynamics and Beyond, Oxford University Press, Oxford, 2001.

[14] G. Hazi and A. Markus, On the bubble departure diameter and release frequency based on numerical simulation results, Int. J. Heat Mass Transfer, 52 (2009), 1472-1480.

[15] A. K. Gunstensen, D. H. Rothman, S. Zaleski, and G. Zanetti, Lattice Boltzmann model of immiscible fluids, Phys. Rev. A., 43 (1991), 4320-4327.

[16] X. Shan and H. Chen, Lattice Boltzmann model for simulating flows with multiple phases and components, Phys. Rev. E., 47 (1993), 1815-1819.

[17] M. R. Swift, W. R. Osborn, and J. M. Yeomans, Lattice Boltzmann simulation of nonideal fluids, Phys. Rev. Lett., 75 (1995), 830-833. 
[18] X. He, S. Chen, and R. Zhang, A lattice Boltzmann scheme for incompressible multiphase flow and its application in simulation of Rayleigh-Taylor instability, J. Comput. Phys., 152 (1999), 642-663.

[19] T. Inamuro, T. Ogata, S. Tajima, and N. Konishi, A lattice Boltzmann method for incompressible two-phase flows with large density differences, J. Comput. Phys., 198 (2004), 628-644.

[20] J. S. Rowlinson and B. Widom, Molecular Theory of Capillarity, Clarendon Press, 1989.

[21] Y. Sone, Asymptotic theory of flow of rarefied gas over a smooth boundary II, In Rarefied Gas Dynamics, ed. D. Dini, (Editrice Tecnico Scientifica, Pisa), 2 (1971), 737-749.

[22] T. Inamuro, Lattice Boltzmann methods for viscous fluid flows and for two-phase fluid flows, Fluid Dyn. Res., 38 (2006), 641-659.

[23] A. J. Briant, P. Papatzacos, and J. M. Yeomans, Lattice Boltzmann simulations of contact line motion in a liquid-gas system, Philos. Trans. R. Soc. Lond. A, 360 (2002), 485-495.

[24] A. J. Briant, A. J. Wagner, and J. M. Yeomans, Lattice Boltzmann simulations of contact line motion. I. Liquid-gas systems, Phys. Rev. E, 69 (2004), 031602.

[25] J. W. Cahn, Critical point wetting, J. Chem. Phys., 66 (1977), 3667-3672.

[26] D. Bhaga and M. E. Weber, Bubbles in viscous liquid: shapes, wakes and velocities, J. Fluid Mech., 105 (1981), 61-85.

[27] T. Inamuro, M. Yoshino, H. Inoue, R. Mizuno, and F. Ogino, A lattice Boltzmann method for a binary miscible fluid mixture and its application to a heat-transfer problem, J. Comput. Phys., 179 (2002), 201-215.

[28] W. Fritz, Berechnung des Maximalvolumens von Dampfdampfblasen, Phys. Z., 36 (1935), 379-384. 